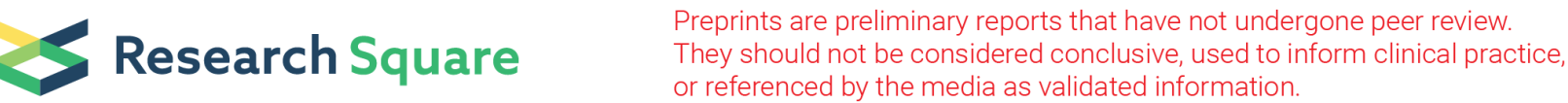

\section{Maternal and Child Health Implementation Research in Mozambique: Effective Community Interventions to Promote Sexual and Reproductive Health}

\section{Paulo Das Das Neves Pires ( $\nabla$ druidatom@gmail.com )}

Lúrio University https://orcid.org/0000-0002-2586-9955

\section{Martins Mupueleque}

University Mussa Bin Bique

David Zakus

University of Toronto

Jaibo Mucufo

Lúrio University

Ahmed Abdirazak

Lúrio University

\section{Cynthia Macaringue}

Lúrio University

\section{Ronald Siemens}

University of Saskatchewan Health Sciences Library: University of Saskatchewan

\section{Celso Belo}

Lúrio University

\section{Research}

Keywords: child health, community, family planning, health services, implementation research, maternal health, Mozambique, participation, pregnancy, reproductive and sexual health

Posted Date: October 21st, 2020

DOI: https://doi.org/10.21203/rs.3.rs-93204/v1

License: (c) (i) This work is licensed under a Creative Commons Attribution 4.0 International License. Read Full License 


\section{Abstract}

Background: High maternal and neonatal mortality rates in Mozambique, are due to adolescent pregnancies, difficulties in accessing health services, traditional constraints, and gender inequalities. An implementation research project, Alert Community to Prepared Hospital in Natikiri, Nampula, Mozambique was developed to reduce maternal and new-born mortality. From 2016 to 2020, it implemented activities to improve population knowledge and function in sexual and reproductive health, and to enable community participation in maternal health services. In this paper we will assess and discuss the impact of community participation on improving sexual and reproductive health.

Methods: Implementation research with community intervention and programmed mid-term evaluations, using mixed methods research, with descriptive quantitative surveys and qualitative focus groups discussions and interviews, applied from 2017 to 2020. Local health committees, traditional birth attendants, traditional healers and local leadership all participated: trained in sexual and reproductive health and participated in radio discussion groups; community and hospital members of the comanagement committee enabled local programming. Maternal and child health indicators were evaluated with health unit's operational data. Quantitative data were captured in Microsoft Office Excel, analysed with SPSS 21 to find frequency, percentage, mean and standard deviation; qualitative data registered in Word was analysed with NVIVO. This research received bioethical approvals from both the Mozambique and Canadian universities and followed Helsinki Declaration recommendations.

Results: Comparing changes from 2016 to 2019, the number of health committees operating in Natikiri rose from 7 to 20. Each committee integrated four Family Health Champions, who attained 24738 residents with health education interventions on reproductive health. A theatre group developed dramas about the same key messages, presented in communities. Population access to contraceptives was facilitated, from $42 \%$ to $91 \%$ in women and from $65 \%$ to $90 \%$ in men. At Marrere Health Centre, women with four ante-natal visits rose by $185 \%$, and children less than one year of age' visits $89 \%$; at Marrere General Hospital deliveries rose $60 \%$.

Conclusion: Community participation, at all levels of maternal and child health service care continuum, from community to hospital, enhanced with complementary interventions well contextualised, is effective in improving adolescent and adult sexual and reproductive health.

\section{Trial registration}

This study was not registered in any data base.

\section{Plain English Summary}

Alert Community to a Prepared Hospital implementation research project was carried out in Natikiri, Nampula, Mozambique, from 2016 to 2020, aiming to reduce maternal and neonatal mortality rates, a public health problem in this country. It deployed activities to improve population knowledge and function 
in sexual and reproductive health and enable community participation in maternal health services: trainings, radio discussion groups, drama.

Using mixed methods research to evaluate results with local health committees, traditional birth attendants, traditional healers, and local leadership we compared maternal and child health indicators evolution at health units.

Comparing changes from 2016 to 2019 , the number of local health committees rose, attaining 24738 residents with health education interventions, population access to contraceptives was facilitated, from $42 \%$ to $91 \%$ in women and from $65 \%$ to $90 \%$ in men, women with four ante-natal visits rose by $185 \%$, children less than one year of age' visits $89 \%$ and maternity $60 \%$.

Community participation at maternal and child health service care demonstrated to be effective in improving adolescent and adult sexual and reproductive health.

\section{Introduction}

Mozambique has high maternal mortality rates $(452 / 100000$ live births, 2017$)$ and child mortality (67.3/1000 live births), ${ }^{1}$ due to a variety of factors including adolescent pregnancies, a high fertility rate, low family planning (FP) practice, insufficient numbers of qualified health professionals (HP), lack of medical equipment and materials, a poor referral system, difficulties in accessing health services (transportation, long waiting times, and illicit charges at the pharmacy to get drugs, in the emergency room to get faster attendance, and in maternity to get delivery support), traditional religious constraints and societally embedded gender inequalities. ${ }^{2,3}$

A participatory implementation research project, ${ }^{4}$ Alert Community to Prepared Hospital care continuum (ACPH), in Natikiri, Nampula, Mozambique, was developed to reduce maternal and new-born mortality, and was subsequently implemented, funded by the International Development Research Centre (IDRC in Ottawa with support from the Canadian Institutes of Health Research and Global Affairs Canada). It took place from 2016 to 2020, including research teams from Lúrio University (UniLúrio, in Nampula) and the University of Saskatchewan (in Saskatoon, Canada).

A base line study was conducted in 2016 , with a large community consultation, including Local Health Committees (LHCs) and other community-based organization members, traditional birth attendants (TBAs), traditional healers and local leadership, with a simple question: "why are pregnant women and new-borns dying in your community?". ${ }^{5}$ Some of the most common answers were complaints about HPs misbehaviour, including illicit charges. The complaint process was unusable at the hospital: community members informed that complaints did not have any impact, and never led to any changes in service delivery. LHCs in Natikiri were mostly inactive, and the hospital co-management committee (CMC) only met occasionally with few participants. 
Strategies were designed to improve population empowerment in sexual and reproductive health (SRH), and enhance access and enable community participation in maternal and child health (MCH) services, an efficient proven strategy, ${ }^{6}$ at Marrere Health Centre (MHC) and Marrere General Hospital (MGH), the health units (HUs) serving the Natikiri population (over 56000).

We based all our objectives on Ministry of Health (MISAU) defined strategies, programs, and tools, and worked in close partnership with the community. MISAU targets illicit charges ("petty" / administrative, bribery, favouritism) ${ }^{7}$ and the issue of difficult access to health care in three ways:

- Development of LHCs, to inform, educate and discuss with community members, regarding health issues.

- Development of health centres and hospitals CMCs, gathering HPs and members of LHCs to discus, inform and cooperate on questions of facility management and provision of health care services.

- Development of the HUs challenges survey tool, ${ }^{8}$ which uses a community and hospital-based report, and meetings to monitor and evaluate hospital function and user satisfaction.

MISAU has designed an effective community participation program, widely known to help increase the quality of public health services, ${ }^{9}$ but this strategy is rarely applied in the field.

To proceed with interventions, we established several partnerships: with MISAU, Nampula Provincial Health Directorate, Nampula City Health Directorate; and several non-governmental organizations, working on health issues in the area.

In this paper we will assess and discuss the impact of our community participation interventions and alliances, on improving SRH, FP and child health in rural northern Mozambique.

\section{Materials And Methods}

The aim of this study was to evaluate the impact of community $\mathrm{MCH}$ empowerment, to better access and quality of $\mathrm{MCH}$ care services in Nampula, Mozambique.

\section{Design}

This is an implementation research with community interventions and programmed mid-term evaluations, using mixed methods research to collect data, with quantitative documental review and descriptive cross-sectional surveys, and qualitative focus groups discussions and interviews, applied from 2017 to 2020 in Natikiri.

\section{Interventions}

To achieve behaviour changes, between 2017 and 2019, we applied a mass media campaign, ${ }^{10}$ and several trainings on SRH, FP, child health, nutrition, community service, were organised and held in the 
local language (Emakhuwa), with all community groups, as recommended in other studies, ${ }^{11}$ together with interventions at HUs (HPs trainings, ante-natal visits and maternity equipment and consumables provision), and monitored by pre and post intervention surveys, focus group discussions and documentary data collection.

We revived and trained other LHCs that have been inactive for several years, promoting their participation in MGH CMC meetings. Each LHC selected and incorporated "family health champions" to visit families with key health education messages, a recognised tool to better mother's health. ${ }^{12}$

A theatre group was formed and created four original short dramas about the same key messages, and subsequently presented them twice monthly in different communities.

Radio discussion groups debated newly developed and diffused SRH weekly broadcasts on radio stations, using the local language, an efficient strategy applied in other countries in Africa. ${ }^{13}$

TBAs, after training, mentored and followed pregnant women through to maternity delivery and new-born visits.

A newly developed local transport system was implemented to take women (and other emergencies) to the maternity at MGH. Six motorcycle ambulances were specifically designed and manufactured, and then were deployed and placed in geographically strategic communities LHCs, covering a total of 18 Natikiri communities. Drivers were licenced and trained in basic first aid and public service, and community-based keepers were selected and trained.

The members of MGH CMC took a leading interest, meeting every two months to review complaints, enabling all aspects of local programming, and voluntarily participating in information and education programs. We facilitated MGH as the first hospital in Mozambique to systematically use the HUs Challenges Survey tool, by the $\mathrm{CMC}$ in regular meetings, monitoring health services evolution and communities' opinions.

This was part of creating a 'prepared hospital' but more so, various specific interventions were directed to the hospital, including construction of a new surgical suite for caesarean sections, other equipment including two ultrasound machines and other supplies, and much provider training through workshops which were all evaluated.

\section{Data collection}

The surveys were carried out on a regular basis, in the catchment area of $\mathrm{MGH}$ and $\mathrm{MHC}$ (both adjacent to UniLúrio), by UniLúrio Faculty of Health Sciences (FHS)' students duly trained, to identify, assess and track changes in relevant community knowledge, attitudes and behaviours about SRH, FP and child health. 
Periodic focus group discussions with each major stakeholder group, were conducted to explore intervention barriers and successes.

Monthly traditional birth attendant reports and motorcycle ambulances activities were tallied and assessed.

Furthermore, to assess the acceptability, appropriateness, and feasibility of the implementation strategies, we carried out in-depth telephone interviews with community members, including adolescent and adult men and women.

Data from MGH and MHC statistics departments was collected periodically.

\section{Data analysis}

Quantitative data were captured in Microsoft Office Excel and analysed with SPSS21, using frequency, percentage, mean, standard deviation.

$\mathrm{MCH}$ indicators were evaluated along with hospital and health centre operational data as was implementation of the project's key messages.

Qualitative data were recorded and transcribed, before being analysed with NVIVO software using a content analysis approach.

\section{Bioethics}

Before implementation, this research received bioethical approvals from both Mozambique and Canadian universities. All participants signed or recorded and informed consent term. The study followed Helsinki Declaration (2013) recommendations.

\section{Results}

Much data was collected and assessed comparing changes from 2016 to 2019.

The number of LHCs actively operating in Natikiri rose from 6 to 20, to cover $95 \%$ of communities and target population; and LHCs members rose from 70 to 337, with health information and education activities reaching more than 10000 persons per year. Each LHC selected and integrated four Family Health Champions (both genders, two adolescents and two adults), and managed to attain 3008 health education interventions with 24738 residents, sharing and discussing SRH key messages in the local language.

The theatre group presented the dramas about the same key messages twice monthly in different communities (public audience estimated at 1600 in 2018 and 1200 in 2019).

Population knowledge scores on family planning (what is it, how to do it and where to get contraceptives) rose from $64-83 \%$ in men and $86 \%$ in women; access to contraceptives was facilitated, from $42-91 \%$ in 
women and from $65-90 \%$ in men. ${ }^{14}$ In 2019 , asked if they wanted to use family planning, $70 \%$ of respondents answered positively but only $47 \%$ of adults and $18 \%$ of adolescents stated they use family planning.

Among the communities the number of trained TBAs rose from 16 to 89, and they assisted 915 pregnant women in 2018 and 2004 in 2019. The number of pregnant women referred to the hospital for delivery rose from 59 (2016) to 708 (2019), and the number of pregnant women with obstetric risk signs being assisted rose from 0 (2016) to 108 (2019).

The motorcycle-ambulance transportation system implemented in six LHCs, and supported in part by newly formed local saving groups, carried 156 (2018) and 122 (2019) women to deliver at MGH maternity, where a significant investment from the project helped improve delivery facilities and personnel.

At MHC, the number of women with a first ante-natal consultation rose 27\% (from 1882 in 2016 to 2393 in 2019); women with four ante-natal visits rose 185\% (from 405 to 1155); and children less than one year of age visits rose $89 \%$ (from 1132 to 2139 ). At MGH maternity deliveries rose $60 \%$ (from 1243 to 1991) during the same period.

The MGH-CMC met 13 times over two years (2018-2019), gathering 68 different LHCs members (TBAs, traditional and religious leaders), and 39 HPs (including the management team, MGH board, statisticians, doctors, and nurses), changing the previous limited representation from the communities, occasional meetings and low impact on MGH and MHC services delivery. Subsequently, this increased volunteer participation in patient orientation, care and cleaning activities (community volunteers were scheduled weekly to be at the hospital to support, inform, and educate users), it reduced the practice of charging illicit fees (and when they did happen, the complaints got to MGH management board and were quickly solved), and helped resolve dysfunctional human resource situations. This all resulted in better service delivery. The $\mathrm{CMC}$ also successfully raised further external funds and started construction on a "Mothers waiting house", outside the MGH, to promote and facilitate deliveries at the maternity.

The interviews revealed that the community believed the project' strategies to be acceptable, appropriate, and feasible. The main themes that emerged from the qualitative analyses included the perceived beneficial nature of the interventions, and their ability to improve knowledge and attitudes about SRH. When asked about the viability of this project, one of our respondents said:

"It is viable, yes, we cannot stop! We have to continue giving lectures in the communities." (Traditional Leader, male, 46 years).

\section{Discussion}

Community participation through empowerment, ${ }^{15}$ is known to be essential to better health services, but requires material and other inputs. ${ }^{16,17}$ When approached by trusted partners, communities are eager to 
decrease maternal and child morbidity and mortality. They volunteer to cooperate, and their leaders are willing to receive health training and are ready to participate in health education in their communities. ${ }^{18}$ The communities were incredibly happy with the local theatre and radio programs.

LHCs are strong preventive medicine and screening agents, able to participate in HUs and services management, and community education.

The CMC intervention had a low cost (over two years we estimated \$900 USD), but was effective at improving health services and decreasing illicit charges, with leadership and monitoring support.

The project's volunteer family health champions, TBAs training programs, community theatre dramas, radio programs and engagement with adolescent initiation rite leaders, increased the local population's knowledge about SRH and rights, leading to more positive attitudes towards FP and hospital deliveries. These achievements have been demonstrated in other low and middle-income countries. ${ }^{19,20,21}$

FP practice remains lower than possible and desirable due to economic constraints, religious and supply barriers.

TBAs became readily engaged as maternal health promoters and were recognised to be excellent referrers and protectors of mothers and babies, as seen in other studies. ${ }^{22}$ They were also found to be appropriate and accepted companions during the hospital deliveries (which was another interesting innovation); similar to evidence presented from other studies in Africa. ${ }^{23}$

The transportation system facilitated many transfers though remained under used, due to technical difficulties (legal, mechanical and communication), and in need of management improvements. Subsequently, during the last year of the research, external funding was procured to enable a social enterprise (Project Frango) to be initiated to assist the sustainability of this transport system.

Facing a financial and human resource crisis common in the public sector, health service performance, nonetheless, improved, sustaining an acceptable quality, and acquiring additional human and material resources. The local population recognised the good performance of MGH and MHC.

The overall emerging increased inclusivity, empowered community leaders and TBAs, to share their SRH and FP knowledge with their communities and improved their attitudes and practices.

Implementation participatory research method, has shown a positive effect to empower population, in terms of health knowledge, attitude and participation in health services management. ${ }^{24}$

\section{Conclusions}

This study shows that community empowerment and participation, at all levels of $\mathrm{MCH}$ service care continuum, from community to hospital, enhanced with complementary interventions well contextualised, 
are acceptable, feasible and effective in improving adolescent and adult $\mathrm{SRH}$, in reducing maternal and new-born morbidity and making significant community contributions.

To reduce maternal and child morbidity and mortality in Mozambique, we need to focus more investment on health information and education, by supporting trusted local partners, to help with the development and maintenance of LHCs, using the local language. These community partners can map, identify, and develop LHCs to strengthen implementation of national policies and strategies, to improve $\mathrm{MCH}$ and the quality of delivery services. It is necessary to create, monitor, and support CMCs in all health centres and hospitals, using the HUs Challenges Survey Tool, to improve the complaints process, reduce illicit charges and improve the overall patient and provider experience.

\section{Abbreviations}

ACPH - Alert Community for a Prepared Hospital care continuum.

CMC - Co-management committee.

FHS - Faculty of Health Sciences.

FP - Family planning.

HP - Health professional.

$\mathrm{HU}$ - Health unit.

IDRC - International Development Research Centre.

LHC - Local health committee.

$\mathrm{MCH}$ - Maternal and child health.

MGH - Marrere general hospital.

MHC - Marrere Health centre.

MISAU - Mozambican Ministry of Health.

$\mathrm{SRH}-$ Sexual and reproductive health.

TBA - Traditional birth attendant.

UniLúrio - Lúrio University.

\section{Declarations}




\section{Ethics approval and consent to participate}

This research was allowed by the Faculty of Health Sciences of Lúrio University, Nampula Provincial Health Directorate, and approved by Lúrio University Bioethics Health Committee (02/CBISUL/16), and the University of Saskatchewan Bioethics Committee (15-112).

We followed all Helsinki Declaration (2013) recommendations, all participants were volunteers, anonymity guaranteed, free to desist if uncomfortable without any negative condition, signing or recording an informed declaration consent term. This research had no risk or remuneration to participants. They agreed to give their time and opinion on this topic to benefit the population, contribute to improve public health policy interventions and implementation research, and empower inhabitants with SRH knowledge.

The study did not involve the use of animals.

\section{Consent for publication}

This manuscript does not contain data from any individual person. Not applicable.

The authors declare they have reviewed this manuscript and agree to submit it to BioMed Central Reproductive Health Journal. The Faculty of Health Sciences at Lúrio University has authorised this publication.

\section{Availability of data and materials}

The datasets used and analysed during the current study are available from the corresponding author on reasonable request.

\section{Competing interests}

The authors declare they have no competing interests with study design or final report, no financial or personal relationships with other people or organizations that could inappropriately influence this research.

\section{Funding}

The study, including base line and interventions, was carried out with funding from the Faculty of Health Sciences of Lúrio University, with the aid of a grant from the Innovating for Maternal and Child Health in Africa initiative - a partnership of Global Affairs Canada (GAC), the Canadian Institutes of Health 
Research (CIHR) and Canada's International Development Research Centre (IDRC), (ALERT COMMUNITY TO PREPARED HOSPITAL CARE CONTINUUM - 108508-001).

\section{Authors contributions}

PP: Study protocol conception and design, data analysis and interpretation, article draft, final approval of the version to be published.

MM: Study protocol design, data treatment, analysis and interpretation, final approval of the version to be published.

DZ: Study protocol conception and design, data interpretation, article draft, final approval of the version to be published.

JM: Study protocol design, data interpretation, final approval of the version to be published.

AA: Study protocol design, data collection and interpretation, final approval of the version to be published.

CM: Study protocol design, data collection and interpretation, final approval of the version to be published.

RS: Study protocol conception and design, data interpretation, article draft, final approval of the version to be published.

CB: Study protocol conception and design, data interpretation, final approval of the version to be published.

\section{Acknowledgements}

Dr. António Falamique, Dr. Delmar Mutereda, Dra. Gizela Bambo, Mr. Carlos Puaheque, Mr. André Kisito; Asimbawe Kiza and members of the Lúrio University Health Research Students Board.

\section{References}

1. Resultados definitivos censo 2017. Instituto Nacional de Estatística. Maputo. 2019. https://www.ine.gov.mz.

2. Estratégia para o Fortalecimento das Intervenções da Parteira Tradicional. Ministério da Saúde. República de Moçambique. Maputo. 2009. https://www.misau.gov.mz.

3. Biza A, Jille-Traas I, Colomar M, et al. Challenges and opportunities for implementing evidence-based antenatal care in Mozambique: a qualitative study. BMC Pregnancy and Childbirth (2015). 15:200. 
4. Loewwenson R, Laurell AC, Hogstedt C, et al. (2014). Participatory action research in health systems: a methods reader. TARSC, AHPSR, WHO, IDRC Canada, EQUINET. Harare. ISBN: 978-0-7974-5976-2.

5. Belo, P. Pires, J. Josaphat, et al. Maternal and new-born mortality: community opinions on why pregnant women and new-borns are dying in Natikiri, Mozambique. International Journal of Research, Volume 04 Issue 6 May 2017. p-ISSN: 2348-6848. E-IAAN: 2348-795x. http://edupediapublications.org/journals/

6. Gilmore B, McAuliffe E. Effectiveness of community health workers delivering preventive interventions for maternal and child health in low- and middle-income countries: a systematic review. BMC Public Health 2013, 13:847. http://www.biomedcentral.com/1471-2458/13/847.

7. Michaud J, Kates J, Oum S. Corruption and Global Health: Summary of a Policy Roundtable. Kaiser Family Foundation. May 2015.

8. Namati Moçambique. Guia de levantamento dos desafios das unidades sanitárias. Ministério da Saúde, República de Moçambique. Maputo. 2017.

9. Minkler M. Linking Science and Policy Through Community-Based Participatory Research to Study and Address Health Disparities. Framing health matters. American Journal of Public Health. Supplement 1, 2010, Vol 100, No. S.

10. Sood S, Shefner-Rogers C, Skinner J. Health Communication Campaigns in Developing Countries. Journal of Creative Communications, 9(1) 83-100. 2014 Mudra Institute of Communications. SAGE Publications. DOI: 10.1177/0973258613517440. http://crc.sagepub.com

11. Horwood C, Butler L, Barker P, et al. A continuous quality improvement intervention to improve the effectiveness of community health workers providing care to mothers and children: a cluster randomised controlled trial in South Africa. Human Resources for Health (2017) 15:39. DOI 10.1186/s12960-017-0210-7.

12. The Health Communication Capacity Collaborative HC3. (2014) Engaging Families for Healthy Pregnancies - A Focused Desk Review of Knowledge, Attitudes and Behaviours Related to Pregnancies in Three High-Risk Situations: Advanced Maternal Age; High Parity; and Rapid Repeat Pregnancies After Abortion or Miscarriage. Johns Hopkins Bloomberg School of Public Health Center for Communication Programs. Baltimore.

13. Pembe A, Urassa D, Carlstedt A, et al. Rural Tanzanian women's awareness of danger signs of obstetric Complications. BMC Pregnancy and Childbirth 2009, 9:12 doi:10.1186/1471-2393-9-12. from: http://www.biomedcentral.com/1471-2393/9/12.

14. Pires $P$, Siemens $R$, Mupueleque M. Improving sexual and reproductive health and practice in Mozambican families with media campaign and volunteer Family Health Champions. Fam Med Com Health 2019;7:e000089. Doi: 10.1136/fmch-2018-000089.

15. Wallerstein $\mathrm{N}$ (2006). What is the evidence on effectiveness of empowerment to improve health? Health Extension Network. World Health Organization Regional Office for Europe. Health Extension Network report). http://www.euro.who.int/Document/E88086.pdf. 
16. Zakus D. Resource Dependency and Community Participation in Primary Health Care. Social Science and Medicine, Vol. 46(4-5), 1998: 475-494.

17. Zakus D, Lysack C. Revisiting Community Participation. Health Policy and Planning, Vol.13 (1), 1998 : $1-12$.

18. Alaofè H, Asaolu I, Ehiri J, et al. Community Health Worker Interventions for Prevention and Management of Diabetes in Low- and Middle-Income Countries: a Systematic Review, Annals of Global Health (2017), https://doi.org/doi:10.1016/j.aogh.2017.10.009.

19. Black R, Taylor C, Arole S, et al. Comprehensive review of the evidence regarding the effectiveness of community-based primary health care in improving maternal, neonatal and child health: 8. summary and recommendations of the Expert Panel. Journal of Global Health. June 2017, Vol. 7 No. 1. 01090. jogh.org. doi: 10.7189/jogh.07.010908.

20. Ahmed S, Creanga A, Gillespie D, Tsui A (2010) Economic Status, Education and Empowerment: Implications for Maternal Health Service Utilizationin Developing Countries. PLoS ONE 5(6): e11190. doi:10.1371/journal.pone.0011190.

21. Desrosiers A, Betancourt T, Kergoat Y, et al. A systematic review of sexual and reproductive health interventions for young people in humanitarian and lower-and-middle-income country settings. BMC Public Health (2020) 20:666. https://doi.org/10.1186/s12889-020-08818-y.

22. Ditai J, Frye L, Durocher J, et al. Achieving community-based postpartum follow up in eastern Uganda: the field experience from the MamaMiso Study on antenatal distribution of misoprostol. BMC Res Notes (2017) 10:516. DOI 10.1186/s13104-017-2849-5.

23. Rokia S, Giani S. Valorisation du rôle des accoucheuses traditionnelles dans la prise en charge des urgences obstétricales au Mali. Dossier spécial : Médecine traditionnelle en Afrique. Ethnopharmacologia, $n^{\circ} 43$, juillet 2009.

24. Pires P. Implementation research methods: an extension tool in health education and information. International Journal of Family \& Community Medicine, Volume 4 Issue 2. MedCrave. 2020. 